\title{
Band inversion at critical magnetic fields in a silicene quantum dot
}

\author{
E. Romera ${ }^{1}$ and M. Calixto ${ }^{2}$ \\ ${ }^{1}$ Departamento de Física Atómica, Molecular y Nuclear and Instituto Carlos I de Física Teórica y Computacional, \\ Universidad de Granada, Fuentenueva s/n, 18071 Granada, Spain \\ ${ }^{2}$ Departamento de Matemática Aplicada and Instituto Carlos I de Física Teórica y Computacional, \\ Universidad de Granada, Fuentenueva s/n, 18071 Granada, Spain
}

(Dated: January 15, 2020)

\begin{abstract}
We have found out that the band inversion in a silicene quantum dot (QD), in perpendicular magnetic $B$ and electric $\Delta_{z}$ fields, drastically depends on the strength of the magnetic field. We study the energy spectrum of the silicene QD where the electric field provides a tunable band gap $\Delta$. Boundary conditions introduce chirality, so that negative and positive angular momentum $m$ zero Landau level (ZLL) edge states show a quite different behavior regarding the band-inversion mechanism underlying the topological insulator transition. We show that, whereas some ZLLs suffer band inversion at $\Delta=0$ for any $B>0$, other ZLLs only suffer band inversion above critical values of the magnetic field at nonzero values of the gap.
\end{abstract}

PACS numbers: 73., 03.65.Vf, 03.65.Pm,

\section{INTRODUCTION}

It is believed that silicene opens new opportunities for electrically tunable nanoelectronic devices 1, 2]. The quantum spin Hall effect [3], chiral superconductivity [4], giant magnetoresistance [5] and other exotic electronic properties have been predicted in silicene.

Silicene takes part of an emerging category of materials called "topological insulators". In these materials, the energy gap $\Delta$ between the occupied and empty states is inverted or "twisted" for surface or edge states basically due to a strong spin-orbit interaction $\Delta_{\text {so }}$ (namely, $\Delta_{\text {so }}=$ $4.2 \mathrm{meV}$ for silicene).

The low energy electronic properties of a large family of topological insulators and superconductors are well described by the Dirac equation [6], in particular, some 2D gapped Dirac materials isostructural with graphene like: silicene, germanene, stannene, etc. Compared to graphene, these materials display a large spin-orbit coupling and show quantum spin Hall effects [7, 8]. Applying a perpendicular electric field $\mathcal{E}_{z}$ to the material sheet, generates a tunable band gap (Dirac mass) $\Delta=\left(\Delta_{z}-s \xi \Delta_{\mathrm{so}}\right) / 2$, with $s= \pm 1$ the spin, $\xi= \pm 1$ the valley and $\Delta_{z}=2 \ell \mathcal{E}_{z}$ the electric potential (see Figure 11). There is a topological phase transition [9] from a topological insulator (TI, $\left|\Delta_{z}\right|<\Delta_{\text {so }}$ ) to a band insulator (BI, $\left|\Delta_{z}\right|>\Delta_{\mathrm{so}}$ ), at a charge neutrality point $(\mathrm{CNP}) \Delta_{z}^{(0)}=s \xi \Delta_{\mathrm{so}}$, where there is a gap cancellation, $\Delta=0$, between the perpendicular electric field and the spin-orbit coupling, thus exhibiting a semimetal behavior. In general, a TI-BI transition is characterized by a band inversion with a level crossing at some critical value of a control parameter (electric field, quantum well thickness [8], etc). In silicene, in absence of boundary conditions, the zero Landau level (ZLL) energy is given by $E_{0}=-\xi \Delta$ and the band inversion at the CNP $\left(\Delta_{z}^{(0)}=s \xi \Delta_{\text {so }} \Rightarrow \Delta=0\right)$ entails a topological phase transition. Actually, the transition in silicene is associ- ated with a non-analytic contribution to the conductivity from the zero Landau level (ZLL) topological edge or surface states, as a result of a band inversion. Indeed, in Ref. 9] it has been shown that the Hall conductivity jumps from 0 to $e^{2} / h$ at the CNP, by tuning the electric field, thus reflecting the transition the from a trivial insulator to a Hall insulator at the CNP. We address the reader to Ref. 9] for further details on the relation between the topological phase transition in silicene and the change in the character of the ZLL at the CNP.

Finite size and boundary conditions on the effective field theory describing these materials brings some extra features [10]. In this letter we study Berry-Mondragon 11] boundary conditions for a circular silicene QD of radius $R$, which introduces some novelties with regard to the previous discussion and leads to additional interesting physical phenomena. In reference [12] the authors studied the energy spectrum of a circular graphene QD with radius $R$ subjected to a perpendicular magnetic field $B$. Here we consider a circular silicene QD subjected to perpendicular magnetic and electric fields, the last one providing a tunable band-gap and introducing new interesting physics with potential applications in nanotechnology.

\section{EFFECTIVE HAMILTONIAN AND EIGENFUNCTIONS}

The low energy dynamics of silicene in the presence of a perpendicular electric field $\mathcal{E}_{z}$ is described by the Dirac Hamiltonian in the vicinity of the Dirac points $\xi= \pm 1$

$$
H_{s}^{\xi}=v\left(\sigma_{x} p_{x}+\xi \sigma_{y} p_{y}\right)-\frac{1}{2} \xi s \Delta_{\mathrm{so}} \sigma_{z}+\frac{1}{2} \Delta_{z} \sigma_{z},
$$

where $\sigma_{j}$ are the usual Pauli matrices, $s= \pm 1$ is the spin, $v$ is the Fermi velocity of the corresponding material (namely, $v=4.2 \times 10^{5} \mathrm{~m} / \mathrm{s}$ for silicene), $\Delta_{\text {so }}$ and $\Delta_{z}$ is the spin-orbit coupling and $\Delta_{z}$ is the electric potential. 


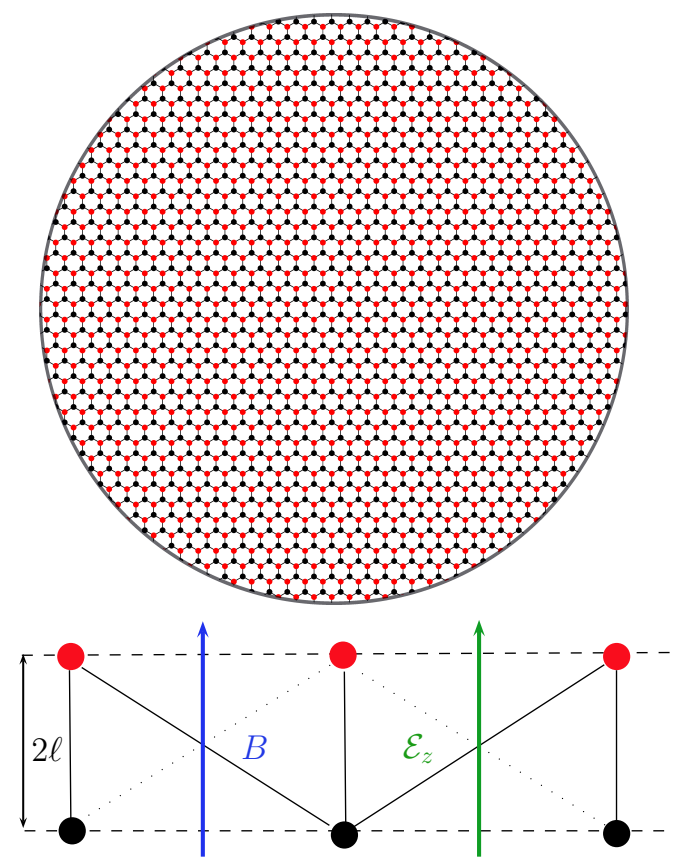

FIG. 1: Illustration of the buckled honeycomb lattice of a silicene quantum dot of radius $R$ in the presence of perpendicular electric $\mathcal{E}_{z}$ and magnetic $B$ fields. Red and black sites form two sublattices separated by a distance $2 \ell$, with $\ell=0.22 \AA$. The lattice distortion (compared to graphene) is due to the large ionic radius of the silicon atoms. The structure generates a staggered sublattice potential $\Delta_{z}=2 \ell \mathcal{E}_{z}$ between red and black sites which provides a tunable band gap $\Delta$.

We shall combine spin-orbit coupling and electric potential into the band gap $\Delta=\left(\Delta_{z}-s \xi \Delta_{\text {so }}\right) / 2$, so that the explicit dependence of $H_{s}^{\xi}$ on the spin $s$ is masked and we can simply write $H^{\xi}$. We also consider a perpendicular magnetic field $B$, which is implemented through the minimal coupling $\vec{p} \rightarrow \vec{p}+e \vec{A}$ for the momentum, where $\vec{A}=B(-y, x) / 2$ is the vector potential in the symmetric gauge. Since $H^{\xi}$ commutes with angular momentum, in order to solve the eigenvalue problem $H^{\xi} \Psi^{\xi}=E \Psi^{\xi}$, we choose energy eigenspinors [we use polar coordinates $(r, \theta)]$

$$
\Psi_{m}^{\xi}(r, \theta)=e^{i \xi m \theta}\left[\psi_{m}^{\xi}(r), e^{i \xi \theta} \chi_{m}^{\xi}(r)\right]^{t}
$$

( $t$ means transpose) which also are eigenstates of angular momentum with eigenvalue $m$ (an integer). The regular solutions at the origin are

$$
\begin{aligned}
\psi_{m}^{\xi}(r)= & \frac{i B \pi v \hbar e^{-\frac{B \pi r^{2}}{4 \phi}}}{(E-\Delta) 2^{\frac{m}{2}-1} r^{m} \phi} \times \\
& {\left[L_{-1-m \tilde{\xi}+a}^{-m}\left(\frac{B \pi r^{2}}{\phi}\right)-\tilde{\xi} L_{-m \tilde{\xi}+a}^{-m-1}\left(\frac{B \pi r^{2}}{\phi}\right)\right], } \\
\chi_{m}^{\xi}(r)= & \frac{e^{-\frac{B \pi r^{2}}{4 \phi}}}{2^{\frac{m}{2}} r^{1+m}} L_{-m \tilde{\xi}+a}^{-1-m}\left(\frac{B \pi r^{2}}{\phi}\right),
\end{aligned}
$$

where $\phi=2 \pi \hbar / e$ is the magnetic Dirac flux quantum, $L_{n}^{m}$ are the associated Laguerre polynomials and we are denoting by $a=\left(E^{2}-\Delta^{2}\right) \phi /\left(B \pi v^{2} \hbar^{2}\right)$ and $\tilde{\xi}=$ $(\xi-1) / 2$. The Berry-Mondragon [11] boundary condition $\chi_{m}^{\xi}(R) / \psi_{m}^{\xi}(R)=i \xi$ at radius $r=R$ provides the characteristic equation for the allowed energies $E$ of the QD.

\section{ENERGY SPECTRUM: ANALYTIC AND NUMERICAL STUDY}

We have numerically solved the Berry-Mondragon boundary condition

$$
\beta_{m}^{\xi}(E, \Delta, B, R)=\chi_{m}^{\xi}(R)-i \xi \psi_{m}^{\xi}(R)=0
$$

and computed the energy $E$ spectrum of a silicene QD of radius $R=70 \mathrm{~nm}$ as a function of the gap $\Delta$ for magnetic field $B=0.1 \mathrm{~T}$ (Figure 2, top panel) and $B=0.6 \mathrm{~T}$ (Figure 2 bottom panel). We have restricted ourselves to angular momentum $m= \pm 3, \pm 2, \pm 1,0$ and valley $\xi=1$. For the valley $\xi=-1$ the results are equivalent swapping $m \rightarrow-m$ and the gap $\Delta \rightarrow-\Delta$.

As we have commented, the topological phase transition in silicene is associated with a non-analytic contribution to the conductivity from the zero Landau level (ZLL). In absence of boundary conditions, the ZLL corresponds to the energy $E=-\xi \Delta[13$ 17], and the band inversion at zero gap $\Delta=0$ entails a topological phase transition. The ZLL still remains in the QD (note the straight diagonal line along the second and fourth quadrants of Figure 2 for $\xi=1$ ), but boundary conditions introduce chirality, which means that positive and negative angular momentum $m$ states have a different behavior. For low magnetic fields, below a critical value $B_{c}=\phi /\left(2 \pi R^{2}\right)$ [see later on eq. (6) for a semiclassical explanation], there only exists a band inversion, (that is, the ordering of the conduction and valence bands is inverted by the tunable band gap which depends on the spin-orbit coupling and the electric field) for positive angular momentum $(m \geq 0)$ ZLLs at $\Delta=0$; all these levels are degenerate with common energy $E=-\Delta$ at valley $\xi=1$ (see Figure 21). Actually, this can also be analytically checked by realizing that $\beta_{m}^{1}(0,0, B, R)$ vanishes only if $m \geq 0$, using properties of associated Laguerre polynomials. On the contrary, negative angular momentum ZLLs detach more an more from the line $E=-\Delta$ as $\Delta \rightarrow-\infty$ (large negative electric field), forming an equally spaced energy band with interlevel spacing of $\epsilon=\hbar v / R$ (they correspond to the energy levels labeled by negative $m$ 's in Figure 21).

In going from $B=0.1 \mathrm{~T}$ (Figure 2, top panel) to $B=0.6 \mathrm{~T}$ (Figure 2 botom panel) we find a band inversion of some negative angular momentum ZLLs at certain nonzero gaps $\Delta_{m}$ (corresponding to given negative electric fields). For the case $R=70 \mathrm{~nm}$, this band inversion starts for the ZLL $m=-1$ at the particular critical magnetic field $B_{c}=\phi /\left(2 \pi R^{2}\right) \simeq 0.136 \mathrm{~T}$. Summing-up, for a 

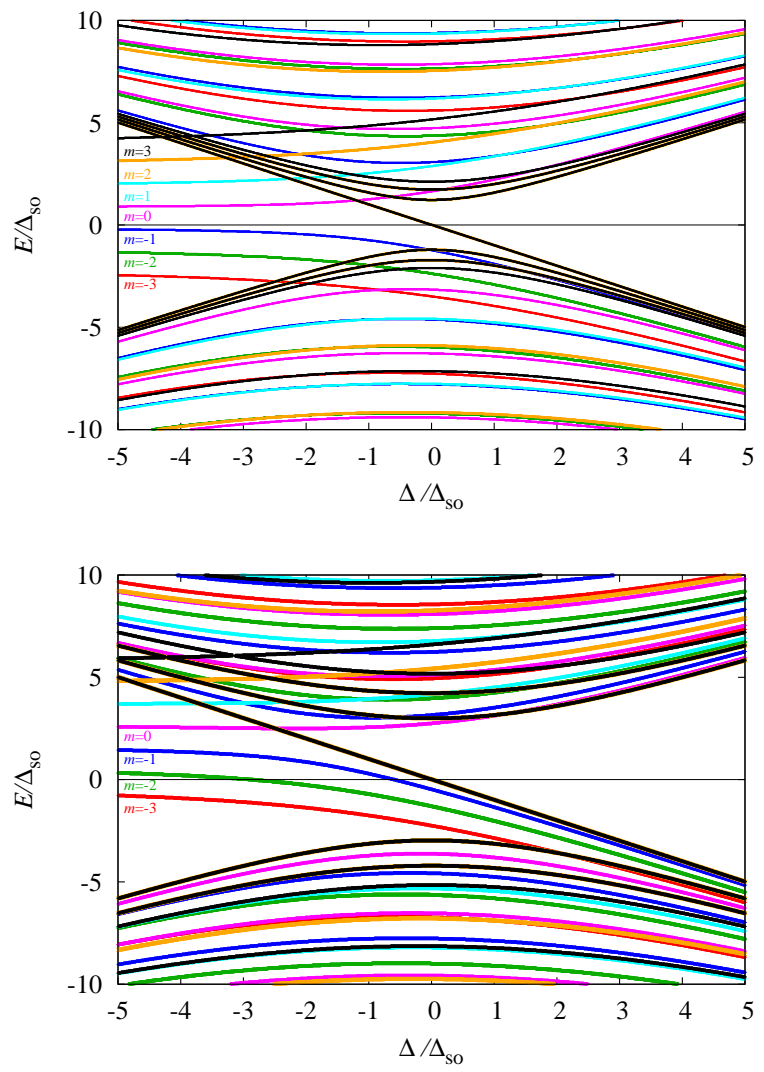

FIG. 2: Low energy spectrum (for angular momenta $m=$ $-3, \ldots, 3$ and valley $\xi=1$ ) of a silicene quantum dot of radius $R=70 \mathrm{~nm}$ in the presence of a perpendicular magnetic field of $B=0.1 \mathrm{~T}$ (top panel) and $B=0.6 \mathrm{~T}$ (bottom panel), bellow and above the critical value $B_{c}=0.136 \mathrm{~T}$, respectively. Energy is given as a function of the gap $\Delta$, which is tunned by applying a perpendicular electric field. Energy and gap are measured in $\Delta_{\text {so }}$ units.

given $R$ and for $B<B_{c}$, there only exists a band inversion for positive angular momentum ZLLs at $\Delta=0$. The situation changes for $B>B_{c}$, when more and more $m<0$ ZLLs become conductive, $E_{m}>0$, at a given gap $\Delta_{m}<0$, for increasing values of the magnetic field. For example, as it can be appreciated in Figure 2 bottom panel, for $B=0.6>B_{c}$, the ZLLs $m=-1$ and $m=-2$ have suffered a band inversion at certain values of $\Delta<0$ (i.e., at certain values of the electric potential $\left.\Delta_{z}=2 \Delta+s \xi \Delta_{\mathrm{so}}\right)$. These states must contribute to the conductivity for these critical values of the magnetic field.

Let us provide a semiclassical argument that explains the aforementioned band-inversion phenomenon for negative angular momentum ZLLs and provides an analytical expression of the magnetic field critical values $B_{c}^{m}(R)$ at which $m<0$ ZLLs suffer a band inversion for a given QD radius $R$. Massless Dirac electrons in silicene make cyclotron motion with frequency $\omega=\sqrt{2} \hbar v / \ell_{B}$ in an external magnetic field $B$, where $\ell_{B}=\sqrt{\phi /(2 \pi|B|)}$ is

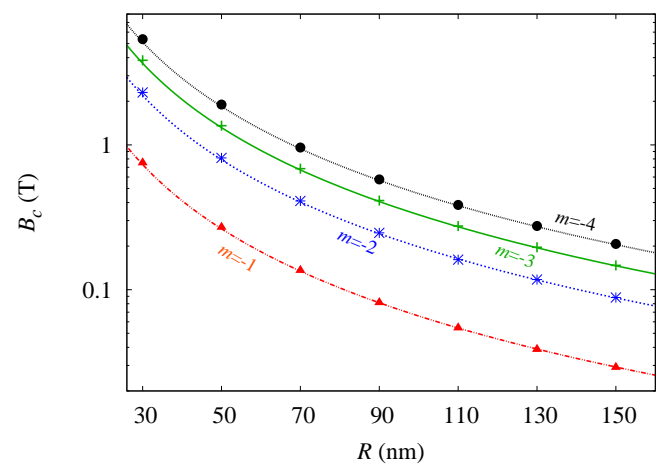

FIG. 3: Critical values of the magnetic field $B_{c}$ (in Tesla), as a function of the silicene QD radius $R$ (in nanometers), at which angular momenta ( $m=-1,-2,-3$ and -4$)$ ZLLs suffer band inversion. Points correspond to the numerical results for $R=30,50,70,90,110$ and $130 \mathrm{~nm}$. Lines correspond to the semiclassical prediction in Eq. [6].

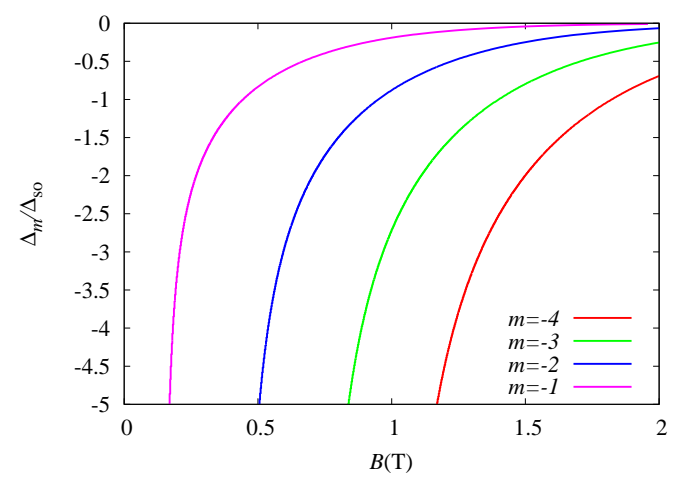

FIG. 4: Gap $\Delta_{m}$ (in units of $\Delta_{\text {so }}$ ) at which there is a band inversion of angular momentum $(m=-1,-2,-3$ and -4$)$ ZLLs, as a function of the magnetic field strength $B$ (in Tesla), for a silicene $\mathrm{QD}$ of radius $R=70 \mathrm{~nm}$.

the magnetic length (the "radius" of the cyclotron motion for the ground state). The probability of finding the electron with angular momentum $m$, at a given radius $r$ in the lowest Landau level, has a sharp peak at $r_{m}=\sqrt{2|m|+1} \ell_{B}$. Is is clear that the corresponding circular trajectory does not fit the QD when $r_{m}>R$ (the QD size). This threshold provides a critical magnetic field depending on $R$ and $m$ given by

$$
B_{c}^{m}(R)=-\xi \frac{(2 m+1) \phi}{2 \pi R^{2}},
$$

where we have also introduced the valley index $\xi= \pm 1$ for completeness. Note that, as we have mentioned before, chiral symmetry is broken, which means that, for positive magnetic fields $B>0$, only negative (resp. positive) angular momentum $m<0$ ZLLs suffer band inversion at valley $\xi=1$ (resp. $\xi=-1$ ) at certain negative (res. positive) values $\Delta_{m}$ of the gap. For negative magnetic fields we have the complementary situation, according to the general formula (6). We have numerically checked 
the semiclassical formula (6) for different negative angular momenta $m$ and $\mathrm{QD}$ radii $R$ at valley $\xi=1$. The band inversion for $m<0$ ZLLs occurs for $B>B_{c}^{m}(R)$ at certain negative gaps $\Delta_{m}(B)$ [see Figures 2 (bottom panel) and 4 . At the critical point $B=B_{c}^{m}(R)$, we have that the energy $E_{m}$ of the $m<0$ ZLL vanishes only for large (negative) electric fields, that is, $\Delta_{m} \rightarrow-\infty$. Therefore, in order to check the prediction (6), we have numerically solved the boundary condition (5) for large (negative) electric potentials and several values of $m$ and $R$. The numerical results (points) in Figure 3 confirm the semiclassical prediction (lines) in Eq. (6) with high accuracy for several $m$ and $R$.

For a given QD size (for example $R=70 \mathrm{~nm}$ ) we have found out that, when the value of the magnetic field increases, there are more and more band inversions of $m<0$ ZLLs at certain gaps $\Delta_{m}<0$ (see Figure 4), corresponding to increasing values of $|m|$ with $m<0$. Moreover, for a given $m<0, \Delta_{m}$ goes to zero as $B$ increases. This calculation has been done by numerically solving the boundary condition (5) for $E=0$ and valley $\xi=1$. We have illustrated this result in Figure 4 for angular momentum ZLLs $m=-1,-2,-3,-4$ and for a silicene QD of radius $R=70 \mathrm{~nm}$.

\section{CONCLUSIONS}

We have studied the energy spectrum of a silicene QD of radius $R$ in the presence of perpendicular magnetic $B$ and electric $\Delta_{z}$ fields, the last one providing a tunable band gap $\Delta$. We have established the existence of critical magnetic fields, given by the semiclassical formula $B_{c}^{m}(R)=-\xi(2 m+1) \phi /\left(2 \pi R^{2}\right)$, above which angular momentum $m$ ZLLs of a silicene QD of radius $R$ suffer band inversion and contribute to the conductivity. Boundary conditions introduce chirality, thus distin- guishing positive and negative angular momentum edge states. When $\operatorname{sign}(m)=\operatorname{sign}(B \xi)$, all angular momentum ZLLs are degenerate, with energy $E=-\xi \Delta$, and all of them suffer a band inversion at gap $\Delta=0$ for any value of the magnetic field. When $\operatorname{sign}(m)=-\operatorname{sign}(B \xi)$ [matching the formula for $B_{c}^{m}(R)$ ], the degeneracy is broken and a band inversion occurs at nonzero gap $\Delta_{m}(B)$ for $|B|>\left|B_{c}^{m}(R)\right|$. As $B$ increases, more and more angular momenta $m$ ZLLs suffer band inversion at gaps $\Delta_{m}(B)$, which go to zero as $B$ increases.

We have performed our calculations in the continuous model, which is a long-wave approximation of the more fundamental tight binding model. Therefore, we have disregarded the effect of lattice termination on the energy spectrum [18]. Nevertheless, we hope that the effect of the boundary irregularities on the spectrum is negligible when the radius $R$ is much larger than the lattice constant, and our results on band inversion at critical magnetic fields remain valid at least in this regime. Of course, a more detailed calculation inside the tight binding framework with more realistic edge termination, like the one done in Ref. 18] for silicene in magnetic field, is necessary to account for the robustness of the band inversion phenomenon. We think that this question deserves a separate study and will be considered elsewhere.

Anyhow, we believe that these critical phenomena in a silicene QD can lead to interesting nanotechnological applications.

\section{Acknowledgments}

The work was supported by the Spanish projects: MINECO FIS2014-59386-P and the Junta de Andalucía projects FQM.1861 and FQM-381. We thank the anonymous referee for bringing to our attention Ref. [18].
[1] P. De Padova et al., J. Phys. Condens. Matter 24, 223001 (2012).

[2] M. Ezawa, Phys. Rev. Lett. 109, 055502 (2012); M. Ezawa, Monolayer Topological Insulators: Silicene, Germanene and Stanene, arXiv:1503.08914

[3] C. C. Liu, W. Feng, and Y. Yao, Phys. Rev. Lett. 107, 076802 (2011).

[4] F. Liu,C-C Liu, K. Wu, F. Yang and Y. Yao, Phys. Rev. Lett. 111, 066804 (2013).

[5] C. Xu, et al., Nanoscale 4, 3111-3117 (2012).

[6] Shun-Qing Shen, Topological Insulators: Dirac Equation in Condensed Matters, Springer-Verlag Berlin Heidelberg 2012.

[7] Kane C L and Mele E J, Phys. Rev. Lett. 95, 226801 (2005)

[8] B. Andrei Bernevig, Taylor L. Hughes and Shou-Cheng Zhang, Science 314, 1757-1761 (2006).

[9] M. Tahir, U. Schwingenschlögl, Scientific Reports, 3, 1075 (2013).
[10] M. Z. Hasan and C. L. Kane, Rev. Mod. Phys. 82, 30453067 (2010)

[11] M. V. Berry and R. J. Mondragon, Proc. R. Soc. London, Ser. A 412, 53 (1987).

[12] S. Schnez, K. Ensslin, M. Sigrist and T. Ihn, Phys. Rev. B 78, 195427 (2008).

[13] V. Yu. Tsaran and S. G. Sharapov, Phys. Rev. B 90, 205417 (2014)

[14] C.J. Tabert and E.J. Nicol, Phys. Rev. Lett. 110, 197402 (2013); C.J. Tabert and E.J. Nicol, Phys. Rev. B 88, 085434 (2013).

[15] M. Calixto and E. Romera, EPL 109, 40003 (2015)

[16] E. Romera and M. Calixto, J. Phys.: Condens. Matter 27, 175003 (2015)

[17] M. Calixto and E. Romera, J. Stat. Mech. (2015) P06029. doi:10.1088/1742-5468/2015/06/P06029

[18] P. Rakyta, M. Vigh, A. Csordás and J. Cserti, Phys. Rev. B 91, 125412 (2015). doi: 10.1103/PhysRevB.91.125412 